\title{
A PRACTICAL APPROACH TO THE CONGENERATION SYSTEM FOR RURAL APPLIANCES
}

\author{
A. Jan Iwaszkiewicz ${ }^{1}$, B. Jacek Perz ${ }^{1}$, C. Jarosław Mikielewicz ${ }^{2}$ \\ 1. The Electrotechnical Institute, Gdansk Branch, Poland \\ 2. The Szewalski Institute of Fluid-flow Machinery, Poland
}

\begin{abstract}
The proposal of distributed generation system based on cogeneration Micro-Power Plants (MPP) is presented in the paper. The proposed MPP can operate using local energy resources as well as traditional fuels. The MPP power range can be from few $\mathrm{kW}$ up to $100 \mathrm{~kW}$. The primeval fuel energy is better utilized in cogeneration MPP than in power plants producing only electricity. The efficiency of cogeneration MPP can reach the level of $90 \%$, while in monogeneration power plants the efficiency is below $50 \%$. 70 to $80 \%$ of the produced energy is used for local heating and 10 to $20 \%$ is used for additional production of electrical energy. The high speed steam turbine using low-temperature evaporation working fluid and an permanent magnet voltage generator directly coupled with turbine are proposed. The different configurations of powerelectronic devices for converting the produced energy to one or three phase $50 \mathrm{~Hz}$ waveforms have been discussed.
\end{abstract}

Key words - renewable energy, distributed generation, cogeneration, power converter.

\section{INTRODUCTION}

The importance of distributed generation systems is still growing nowadays not only within UE but worldwide. They can be based on local energy resources, renewable energy sources and technologies using conventional fuels [1]. For small and medium scale plants popular are multifunctional energy production processes like cogeneration, trigeneration and poligeneration. The cogeneration process, which means simultaneous generating heat and electrical energy, is the most commonly used. The actual energy production development trend, called cogeneration energetic, is based on the distributed low power sources using cogeneration. The European Commission Directive 2004/8/UE defines the target for energy production within UE till a year 2010. The goal is to increase the contribution of small and medium scale cogeneration power plants in energy production up to $18 \%$.

This program creates an unique opportunity for evolution of Micro-Power Plants (MPP). MPP are designed to provide heat and electricity supply for household, farm or small enterprise. The generated heat can be used locally for heating and produced electricity can be used locally or transferred to the power grid, thus lowering the costs of electrical energy supply. Depending on local potential MPP can use conventional or renewable fuel.

The efficiency of power plants producing only electrical energy reaches the level of forty to fifty percent. The primeval fuel energy is better utilized in cogeneration MPP than in power plants producing only electricity. The efficiency of cogeneration MPP can reach the level of $90 \%$. 70 to $80 \%$ of the produced energy is used for heating and 10 to $20 \%$ is used for additional production of electrical energy. It means that the owners of the boilers equipped with MPP can became producers of electrical energy.

Higher efficiency in utilizing the primeval fuel energy in cogeneration MPP results in lower emission of harmful substances produced by process of burning fuel. A small cogeneration MPP can be fully automatically maintained without operating personnel. The marketing study done i.e. in Great Britain showed that the market requirement for Micro-Power Plants can be enormous, in millions pieces, comparable with the requirement for i.e. refrigerators.

Taking into account that the power of such kind of power plants amount from few to hundred $\mathrm{kW}$ it is easy to calculate that they can replace a number of big conventional power plants. If for example the requirement for heating is about $10 \mathrm{~kW}$, it is possible to obtain about $2 \mathrm{~kW}$ of electrical energy, accompanying the heat production. If one million of users took the advantage of this process, the impact on the power system is equivalent to installation of additional big power plant with $2000 \mathrm{MW}$ power. This simple calculation shows how the presented solution can provide for efficient use of primeval energy and enhancement of environment.

There is a number of different concepts of MPP realization using i.e. combustion piston engine, gas turbine, steam turbine, Stirling engine, fuel cells stack and others. Some of these concepts will be put to work in the nearest future. Authors opinion is that in next 2-3 years a cogeneration MPP using steam turbine can reach the market. 
Based on experience and knowledge the authors propose a new concept of the cogeneration steam MPP. The stimulus medium for the steam turbine is low-temperature evaporation fluid of the same kind as used in cooler appliances. This type of medium allows much lower working temperature than combustion engine or gas turbine, thus the materials for turbine construction can be cheaper and the production technology simpler. Thanks to this the cost of electrical energy produced in MPP should be similar to the prices of the energy produced in traditional large power plants.

The range of turbine power for MPP is from few $\mathrm{kW}$ up to hundred $\mathrm{kW}$. The smallest micro-turbines of power $2 \div 10 \mathrm{~kW}$ will be designed for household use, while the bigger ones with power up to $100 \mathrm{~kW}$ will be used in homesteads and small enterprises. Thanks to the use of modern materials and technology the dimensions of MPP are small. The basic idea of the household MPP is presented in fig. 1.

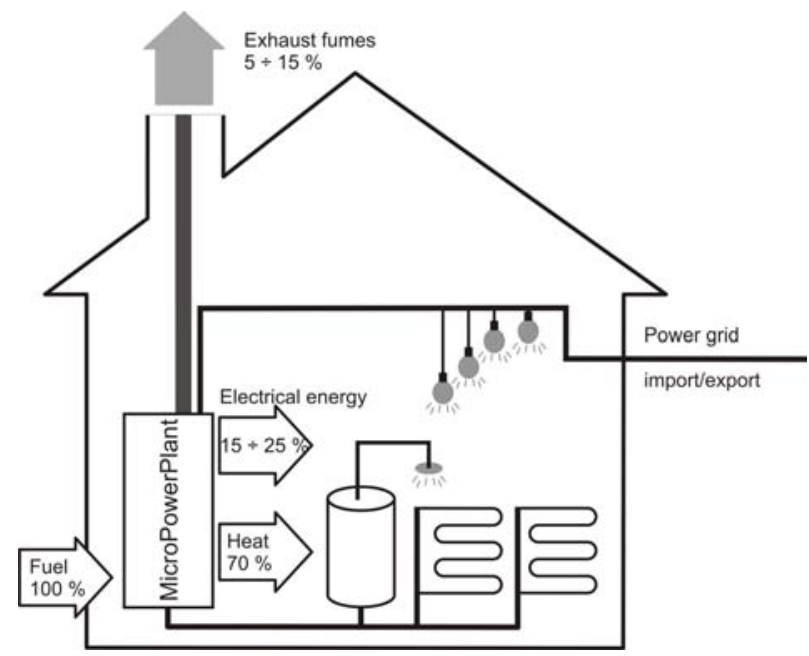

Fig. 1. The basic idea of cogeneration household MPP.

The usage of energy is as following: about $70 \%$ of the fuel energy is used for heating and $15 \div 25 \%$ is converted to electricity. The remaining $5 \div 15 \%$ is lost with exhaust fumes.

The crucial parameter for the energy balance is efficiency of fuel energy conversion to electrical energy. This efficiency consists of micro-turbine and electrical generator efficiency as well as power converter efficiency. If the efficiency of each of the components in this chain was $90 \%$ then the effective efficiency of conversion is only $73 \%$. With the components efficiency of $95 \%$ the efficiency of conversion rise up to $86 \%$. So all the MPP components should be designed with the extreme emphasis on efficiency.

\section{MICRO-TURBINE SYSTEM}

The overview of micro-turbine system is presented in fig. 2 .

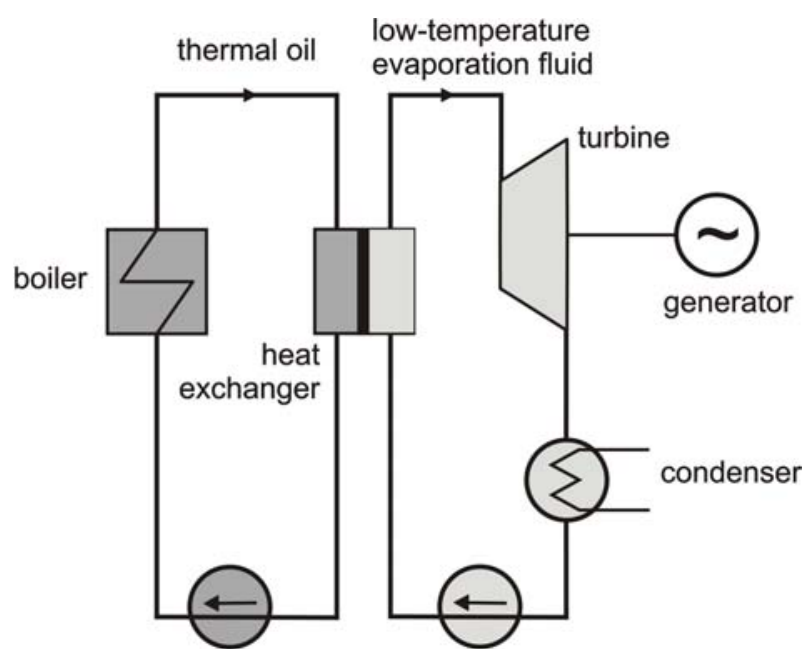

Fig. 2. The overview of micro-turbine system.

The turbine system uses modern Organic Rankin Cycle (ORC) technology, which allows higher parameters than classical water cycle transmission. The main components of the system are: boiler, oil pump, heat exchanger between thermal oil and lowtemperature evaporation working fluid working as a steam generator, micro-turbine, condenser and fluid pump.

For the small MPP with power about $3 \mathrm{~kW}$ a miniature turbine with high rotation speed of about $100000 \mathrm{rpm}$ is considered as an optimal solution. An example of micro-turbine designed by The Szewalski Institute of Fluid-Flow Machinery is shown in fig. 3.

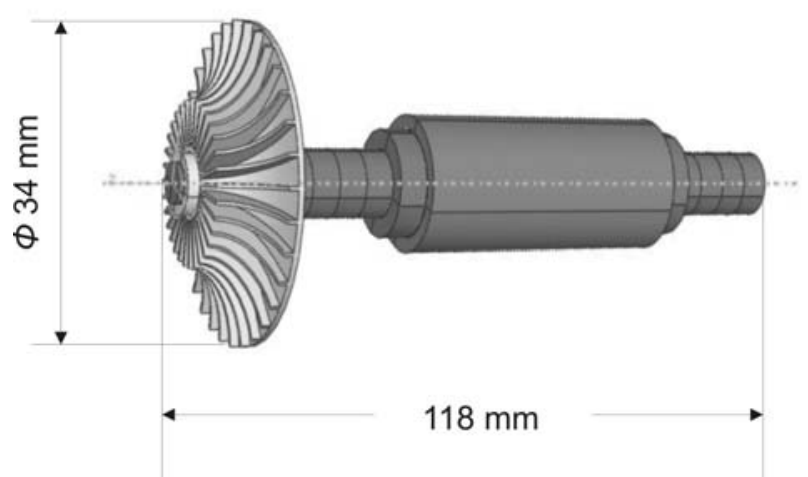

Fig. 3. An example of micro-turbine design.

\section{POWER GENERATOR}

The electrical energy is supplied by voltage generator which should work with the same rotation speed as the turbine. In the relevant literature there are many descriptions of micro-turbine systems equipped with high speed electric generator. For 
higher power range above $250 \mathrm{~kW}$ there are also available economical analyses with prediction of costs per one $\mathrm{kW}$ of produced energy.

In high rotation speed generation systems the permanent magnet machines are used. The construction of generator depends on its power. For the generator of $250 \div 500 \mathrm{~kW}$ the best solution is high machine with axial flux. The construction of this kind of machine [2] is presented in fig. 4. This type of generator can be adapted to the rotation speed of micro-turbine up to $0.5 \mathrm{MW}$ i.e. $30000 \mathrm{rpm}$.

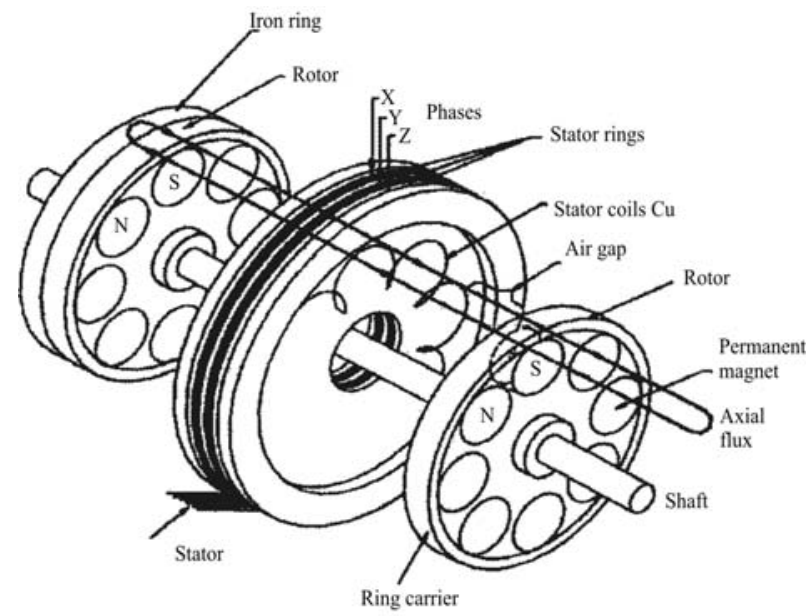

Fig. 4. High speed axial flux generator.

The machine working with this rotation speed generates three phase voltage of frequency $2 \mathrm{kHz}$, which should be converted to three phase sinusoidal waveform 3 x $400 \mathrm{~V}, 50 \mathrm{~Hz}$.

The generator of this construction assures higher power density for volume and weight then classical synchronous generator. The construction of the generator is simple. The magnetic flux is closed by the iron last ring of the rotor.

\section{POWER-ELECTRONICS CONVERTERS}

Thanks to the progress in power-electronics, especially in switching devices technology, it is possible to design converter devices of efficiency in the range $95 \div 98 \%$. The MPP idea assumes that part or all of the produced electrical energy can be delivered to the external power network to be consumed by other users.

The used in MPP high speed permanent magnet machine generates distorted voltage waveforms of frequency much higher then $50 \mathrm{~Hz}$. This chapter presents power-electronics solutions for conversion of generated electrical energy to the usable form with parameters complying with standard EN 50160. The basic properties of the four different solutions will be considered.

\subsection{Diode rectifier converter}

The converter consisting of diode rectifier and voltage inverter is the simplest solution for converting the generated voltage to usable form. The schematic diagram of such converter is presented in fig. 5 .

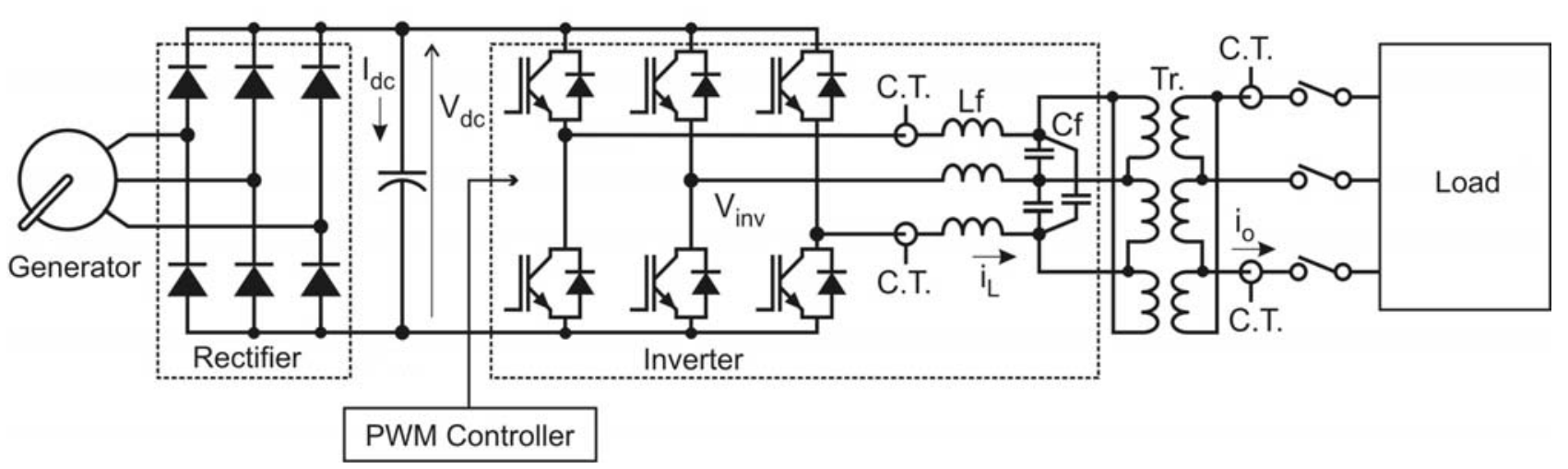

Fig. 5. Schematic diagram of the diode rectifier converter.

Except the three phase diode rectifier and voltage inverter the converter includes also gamma type filter and the transformer. The three phase voltage, generated by turbine driven generator, after rectifying supplies DC intermediary circuit of the inverter. The inverter is built using IGBT (Insulated Gate Bipolar Transistor) switches and works with switching frequency of $3 \div 5 \mathrm{kHz}$. The inverter control is accomplished using digital PWM (Pulse Width Modulation) controller equipped with DSP (Digital Signal Processor) central unit. The output voltage is filtered using gamma type passive filter $\mathrm{L}_{\mathrm{f}}-\mathrm{C}_{\mathrm{f}}$ and applied to transformer $\mathrm{Tr}$, which provides galvanic separation and assures voltage level adjusting. 
The presented converter makes a typical solution creating a link between micro-turbine driven generator and a power network. It assures conversion of the mechanical energy of rotating micro-turbine into electrical energy complying with standards requested by user. This straightforward solution is relatively simple and cheap, but requires a sophisticated control algorithm. The galvanic separation allows parallel connecting of microturbine-converter systems to the common line.

\subsection{Converter with active filter}

The converter with active filter is an expanded modification of the converter described in chapter 3.1. The schematic diagram of such structure is presented in fig. 6 .

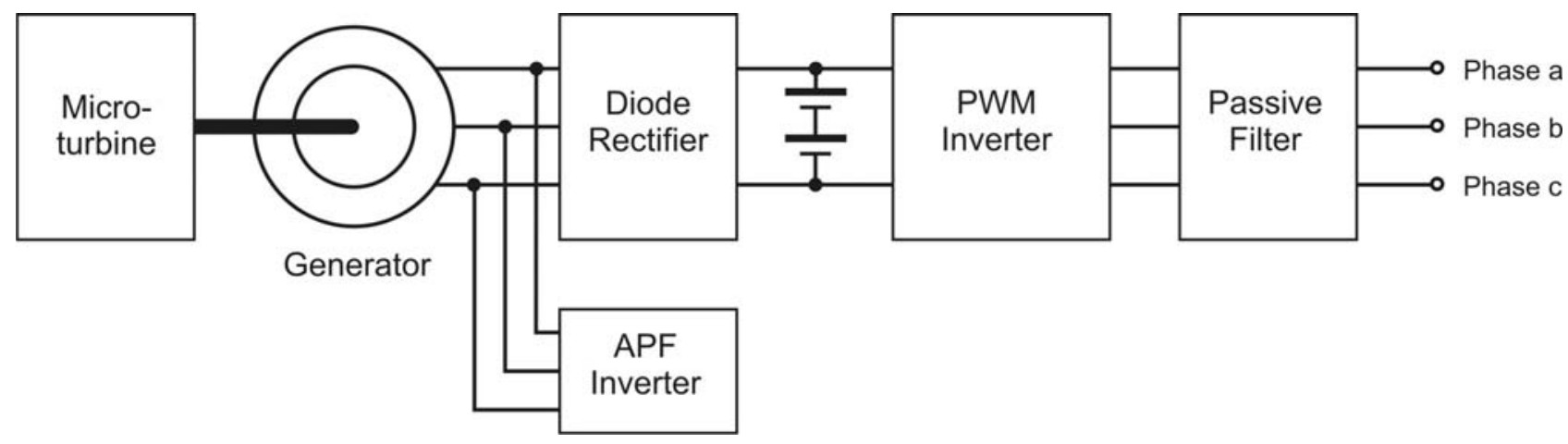

Fig. 6. Schematic diagram of the diode converter with active filter.

As in previous solution the converter consists of the diode rectifier voltage inverter and gamma type filter output filter. The micro-turbine driven generator supplies through the diode rectifier intermediary DC circuit of the voltage inverter. The voltage inverter is built using IGBT or MOSFET transistors and controlled with PWM pattern. An additional APF (Active Power Filter) inverter is connected directly to the generator terminals and is used for the shape enhancement of the generator phase currents.

The active filtration is one of the most dynamic branches of the contemporary power-electronics. It is related to the fact that power-electronic devices, which are nowadays in mass use, create a substantial amount of disturbances in power grid voltages and currents. To limit the scale of the disturbances it became indispensable to introduce special passive or active filtering circuits. The input currents of the rectifiers, which are commonly used in inverters, are violently distorted and they introduce a large amount of harmonics to the supplying network. The typical power-electronics inverter like i.e. presented in fig. 5 is used for converting the input $\mathrm{AC}$ waveforms to $\mathrm{DC}$ voltage, which is then converted back to the output $\mathrm{AC}$, but with different parameters.

In micro-power station application the energy converting process can be described as following:

$$
\operatorname{AC}\left(f_{1}\right) \Rightarrow \mathrm{DC} \Rightarrow \operatorname{AC}\left(f_{2}\right) \text {. }
$$

The frequency of the $\mathrm{AC}$ voltage on the generator output pins is $f_{1}(2 \mathrm{kHz}$ or higher $)$ while the frequency of inverter output voltage should be $f_{2}\left(50 \mathrm{~Hz}\right.$ in Poland) which means that $f_{1}>>f_{2}$. The shape of the high rotation speed generator output voltage is deformed in comparison to the sinusoid. In the same time the nonlinear load of the inverter's rectifier creates large amount of harmonic distortions in output currents of the generator. The distorted load of the generator has a disadvantageous impact on the efficiency and durability of micro-turbine. The active filtering system can significantly improve this situation and increase the efficiency of the whole system. There are many works related to this subject in the literature. For instant the work [6] describes the active filter which after switching the system on accomplishes start of the micro-turbine and works as an active filter after this.

The control system of the inverter working as an active filter should be equipped with the appropriate regulator of the chosen inverter parameters. An example of the regulator solution is presented in the fig. 7. The block named "Non-linear load" represents $\mathrm{AC}\left(f_{1}\right) \Rightarrow \mathrm{DC} \Rightarrow \mathrm{AC}\left(f_{2}\right)$ converter, connected to the phase terminals of the generator. The regulation principle is to stabilize the required level of the DC voltage in the intermediary circuit of the inverter $\mathrm{V}_{\mathrm{DC}} *$. The requested voltage is compared with the current voltage of this circuit and the difference of levels is connected to the input of PI or PID regulator. The voltage regulator in connection with phase currents deviation detector calculates the necessary levels of the sinusoidal phase currents.

In the next chapter the two inverter control methods, which can be applied to the active filter 
control, will be described.

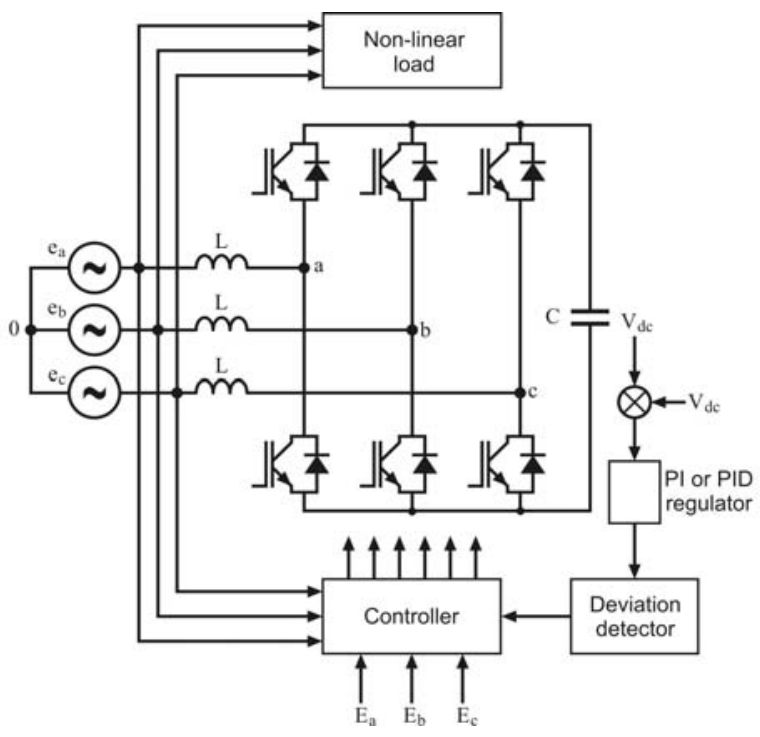

Fig. 7. An example of active filter regulator.

\subsection{Hysteresis control of the active filter}

In last twenty years there have been developed many inverter control strategies based on phase currents deviation. The presented below strategy is taken from [8]. The described predictive control algorithm can be treated as belonging to the family of hysteresis algorithms. The purpose of this algorithm is to keep the instantaneous deviation of the phase current (represented by the current deviation vector) in the defined tolerance range, which complies with the hysteresis algorithm rule.

The prediction mechanism extends the simple algorithm by adding additional procedures for choosing the vector with the longest time of the operation and checking the zero vector switch-on conditions.

The diagram of control system realizing a simple hysteresis algorithm is presented in fig. 8. The controller is equipped with deviation comparators which are periodically switched-on. The algorithm is based on periodical control of the instantaneous values of the phase currents $i_{\mathrm{a}, \mathrm{b}, \mathrm{c}}$. The deviation signals, probed with constant frequency, are connected to the inputs of the analog comparators with the set hysteresis area $\mathrm{H}$. The probing frequency $\mathrm{T}_{\mathrm{p}}$ is chosen appropriately to the dynamic parameters of the controlled object, The hysteresis area is also correlated with the probing frequency and the parameters of the load circuit.

The algorithm will be analyzed using space vector theory. The voltage space vector is defined by its module and position in the complex plane.

The available inverter vectors $\vec{V}_{k}$, electromotive force vector $\vec{e}$ and voltage vector $\vec{U}_{\mathrm{R} 0}=\mathrm{R}_{f} \cdot \vec{I}_{0}$ are presented in fig. 9.

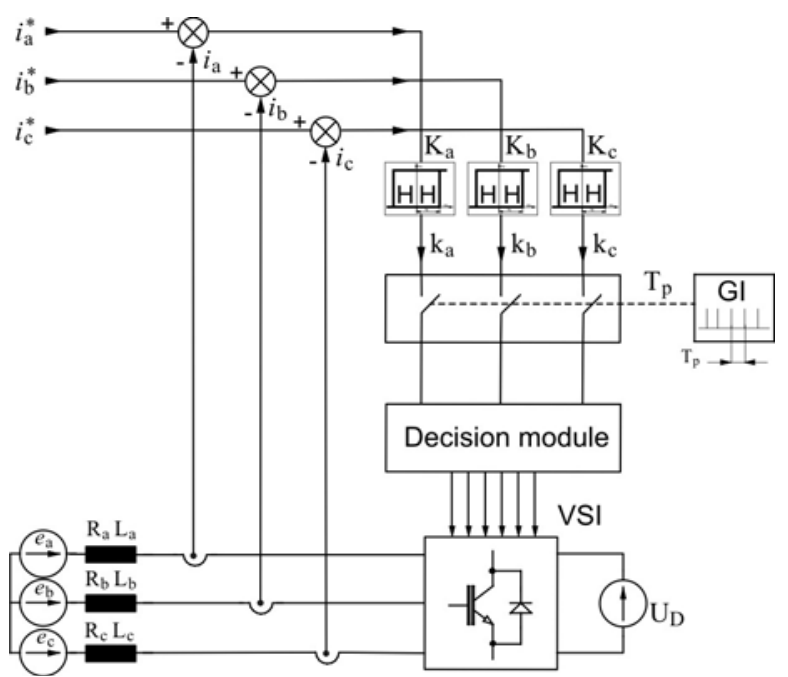

Fig. 8. An inverter control with three periodically switched deviation comparators.

The active vectors $\vec{W}_{k}$ are defined as:

$$
\vec{W}_{k}=\vec{V}_{k}-\left(\overrightarrow{\mathrm{E}}+\overrightarrow{\mathrm{U}}_{\mathrm{R} 0}\right)
$$

where $\overrightarrow{\mathrm{E}}$ is electromotive force vector, $\vec{U}_{\mathrm{R} 0}$ is voltage vector related to the voltage drop on the load resistance and $\vec{V}_{k}$ is inverter output voltage vector. The active vectors $\vec{W}_{k}$ are the voltage vectors forcing the inverter output currents.

The idea of the algorithm will be presented on the basis of the current deviation vector hodograph. The vector hodograph means the trajectory created by the end of the vector in consecutive probing intervals. The definition of the deviation vector $\vec{\delta}$ is as following:

$$
\vec{\delta}=\vec{i}-\vec{i}^{*} \quad \text { where } \quad \vec{i}^{*}=I_{m} e^{\mathrm{j} \omega t} .
$$

It can be proved that the end of so defined current deviation vector is moving along the actually chosen active vector $\vec{W}_{k}$. The permissible area of the current deviation trajectory can be defined i.e. as hexagon limited by the six lines defining the border of the current deviation tolerance for the each phase.

The aim of the current control is to keep the hodograph of the current deviation vector within the defined area. Because of the limitations related to the used digital conversion method, the hodograph of the current deviation vector can cross the border of this area. It should be noted that the instants of passing the borders differ from the current probing periods. 


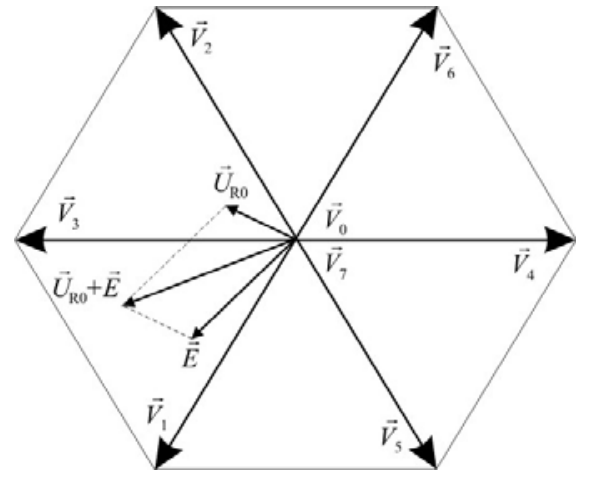

a) space vectors $\vec{V}_{k}, \overrightarrow{\mathrm{E}}$ and $\vec{U}_{\mathrm{R} 0}$.

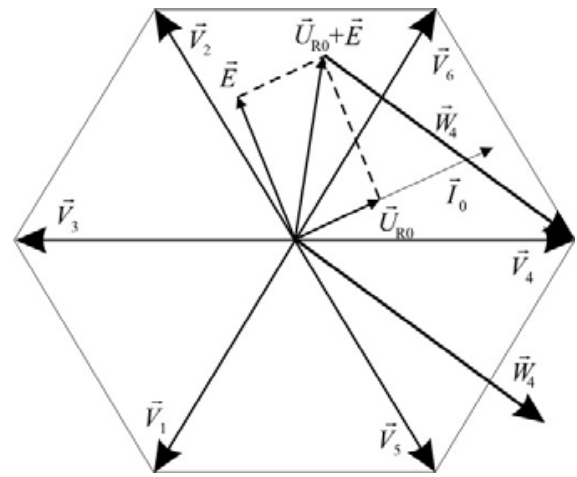

b) construction of the active vector $\vec{W}_{4}$.

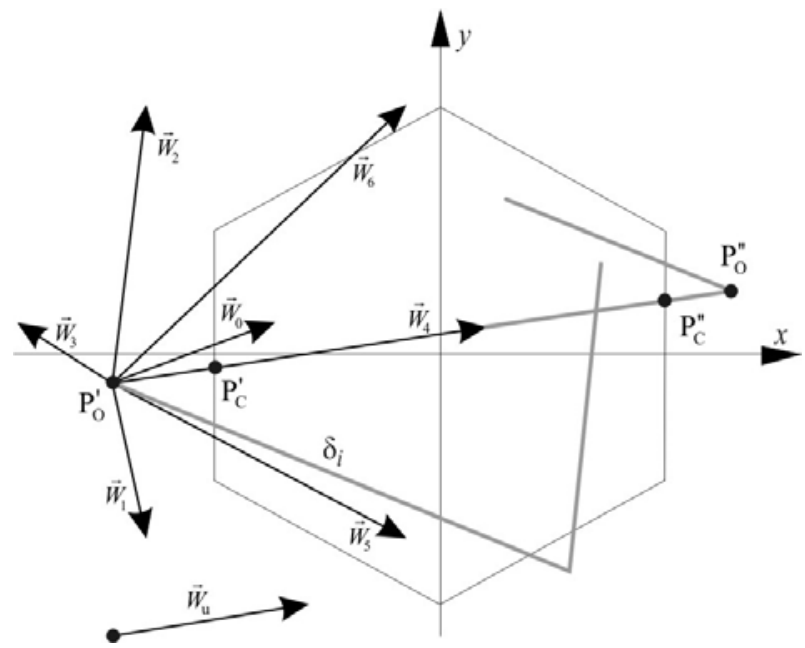

c) active vectors in consecutive probing time.

Fig. 9. Voltage and current space vectors of the inverter.

Fig. 9c presents the situation when during the probing interval the end of the current deviation vector $\mathrm{P}_{0}^{\prime}$ is located outside the requested hexagon. It means that it is necessary to change the inverter output vector. Only four active vectors $\left(\vec{W}_{0}, \vec{W}_{4}, \vec{W}_{5}, \vec{W}_{6}\right)$ can be used to move the end of deviation vector back inside the hexagon. The chosen vector will move the end of the deviation vector in its direction as long as it is switched on. The algorithm will choose vector $\vec{W}_{4}$ because this vector will keep the end of deviation vector inside the hexagon for the longest time. When the end of the current deviation vector will reach point $\mathrm{P}_{0}^{\prime \prime}$, passing the hexagon border once again, the procedure of choosing the active vector will be repeated.

In proposed algorithm the acting time of the chosen active vector is a multiplicity of the probing interval. The active vector acting during one probing interval is presented in fig. 9c as unitary vector $\vec{W}_{u}$. The acting vectors are multiplications of the unitary vectors.

\subsection{Current mode control of the active filter}

The output voltage waveforms of the microturbine driven generator are of approximately constant frequency and amplitude. These properties allow to apply the following current mode control of the active filter.

The assumed phase current of the generator $i_{\mathrm{G}}(t)$ has a shape of the sinusoid:

$$
i_{\mathrm{G}}(t)=I_{\mathrm{G} \max } \sin (\omega t)
$$

and consists of the inverter rectifier input current $i_{\mathrm{I}}(t)$ and active filter current $i_{\mathrm{F}}(t)$ :

$$
i_{\mathrm{G}}(t)=i_{\mathrm{I}}(t)+i_{\mathrm{F}}(t)
$$

It means that the requested phase current of the active filter can be calculated as:

$$
i_{\mathrm{F}}^{*}(t)=i_{\mathrm{G}}(t)-i_{\mathrm{I}}(t)=I_{\mathrm{G} \max } \sin (\omega t)-i_{\mathrm{I}}(t)
$$

The active filter works in this case as a compensator of the current distortion created by the non-linear load of the inverter. The control system generates the requested instantaneous generator current $i_{\mathrm{G}}(t)$ and compares it with the instantaneous value of the inverter rectifier input current $i_{\mathrm{I}}(t)$. To accomplish this task the information the controller needs the following information:

- rotation angle speed of the generator $\omega$,

- actual phase of the generator voltage,

- actual value of the inverter input current.

As an example of the active filter application for trimming the phase currents can be used simulation and experimental works on the complex converter consisting of two inverters: a main one and an auxiliary active filter one.

In this application the active filter is used for improving the shape of the main inverter output phase currents [9]. In fig. 10 the simulation results for this 
type of complex converter are presented. In the upper part of the figure there are required current waveform (2) and one phase output current of the main inverter (1). In the lower part there are required phase current of the filter inverter (3) and load current waveform (4) in the same phase. The frequency of the current was in this case $1 \mathrm{~Hz}$.

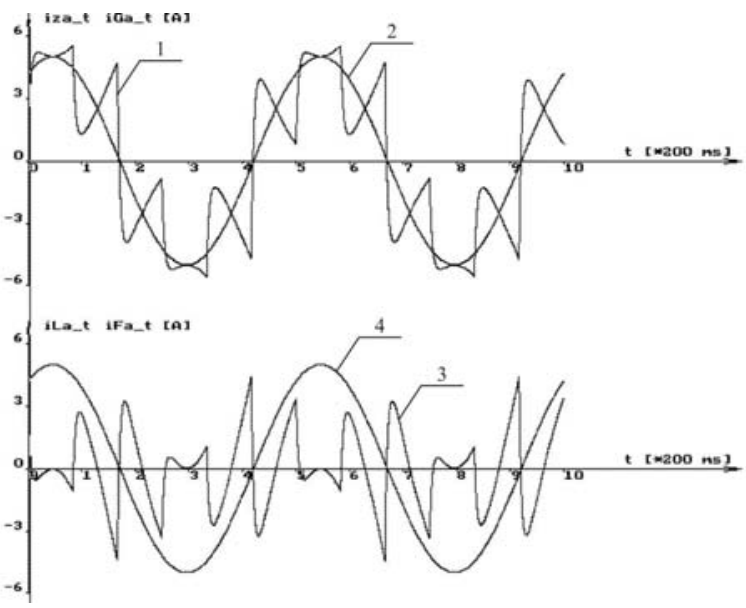

Fig. 10. Simulation of the $1 \mathrm{~Hz}$ current waveforms in one phase of the inverter with active filter.

The experimental verification of these results are presented in fig. 11. R1 is the waveform of the main inverter current, R2 - the waveform of the active filter current, R3 - load current.

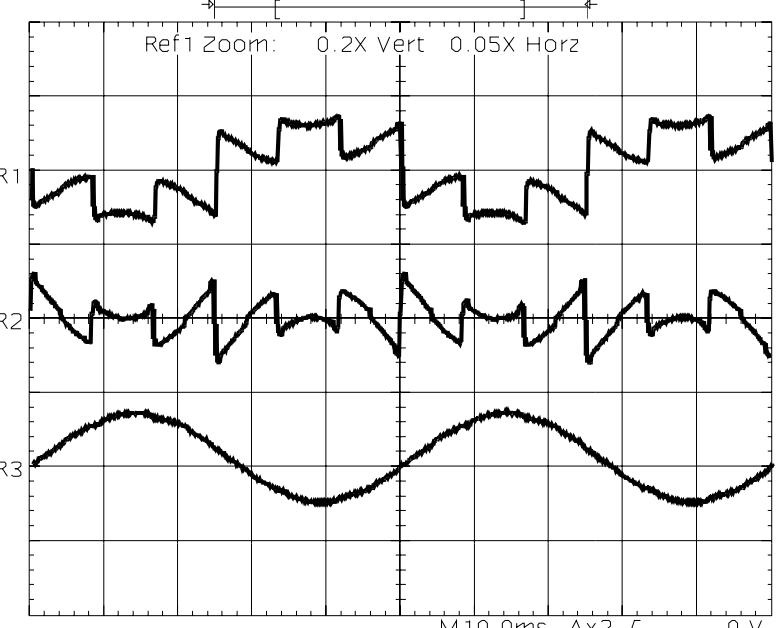

Fig. 11. Experimental verification of the results presented in fig 10 .

A similar set of results for frequency $50 \mathrm{~Hz}$ is presented in fig. 12 and 13.

The presented results prove that the proposed idea of active filter control can be efficiently applied for trimming the shape of the phase currents. The tests have been carried out for current frequencies up to $50 \mathrm{~Hz}$. In case of high rotation speed generator the frequency is higher, but it makes not much difference because the semiconductor switches used in this kind of inverters are able to work on much higher frequencies.

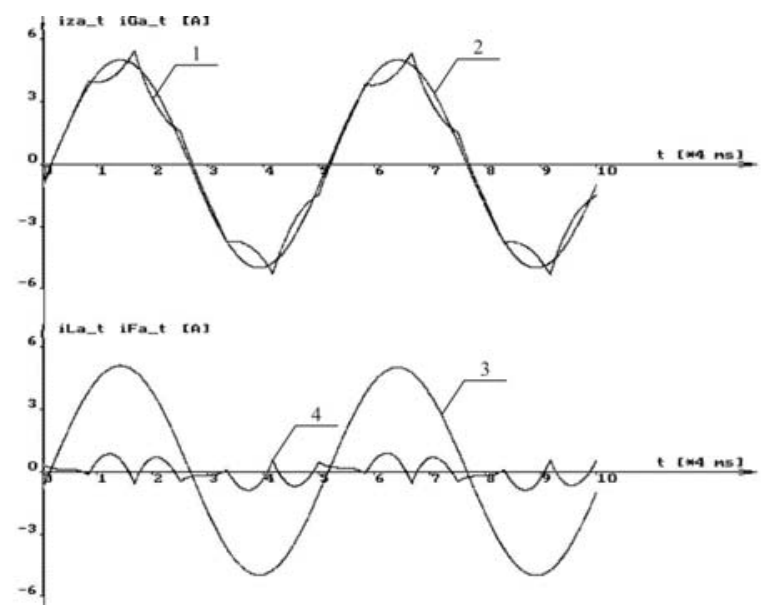

Fig. 12. Simulation of the $50 \mathrm{~Hz}$ current waveforms in one phase of the inverter with active filter.

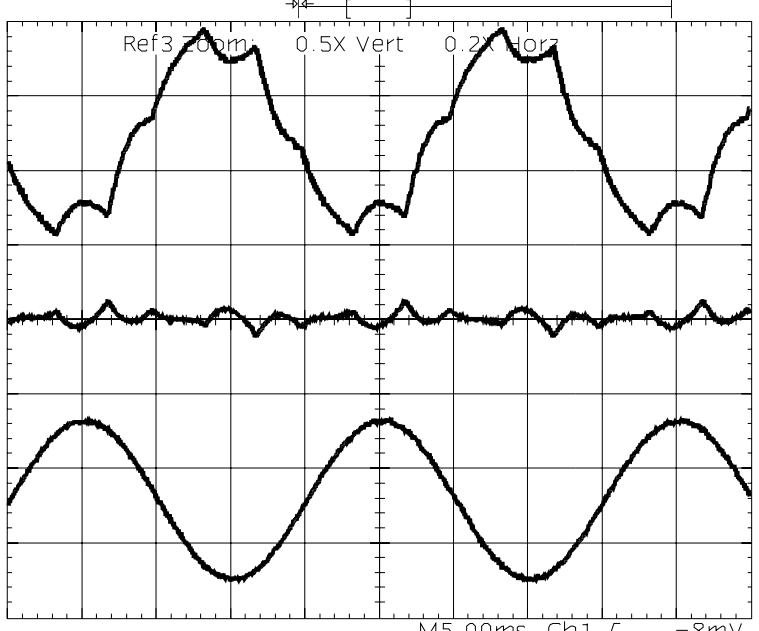

Ref3 $200 \mathrm{mV} \quad 5.00 \mathrm{~ms}$

Fig. 13. Experimental verification of the results presented in fig 12.

\subsection{Matrix converter application}

A schematic diagram of the matrix converter application is presented in fig. 14. The main part of the circuit is two-stage matrix converter. The first part of it works as high efficiency rectifier, while the second part is an inverter generating the grid voltage.

The three-phase voltage from the generator is initially filtered in passive R-L-C filter circuit and then connected to the input of the first stage of the matrix converter, which acts as an "intelligent" rectifier. This kind of rectifier allows for substantial limitation of the passive power $(\cos \varphi=1)$ and improvement of the shape of the phase current waveforms. The advantage of 
this solution is related to the elimination of the electrolytic capacitor from the intermediary DC circuit of the inverter. The electrolytic capacitor, which is indispensable in typical inverter circuitry is the less reliable component of the whole power-electronics system. The proposed rectifier allows for four-quadrant work with the power factor nearing one.

Nevertheless to use this technique it is necessary to develop a control system equipped with powerful DSP processor to realize the sophisticated control algorithm in real time [7].

The rectified voltage from the matrix converter supplies the standard type of the inverter, which task is to convert the electrical energy from $\mathrm{DC}$ to $\mathrm{AC}$ with the parameters required by the power grid. This inverter is controlled using PWM method and its output voltage is filtered using typical passive L-C filter .

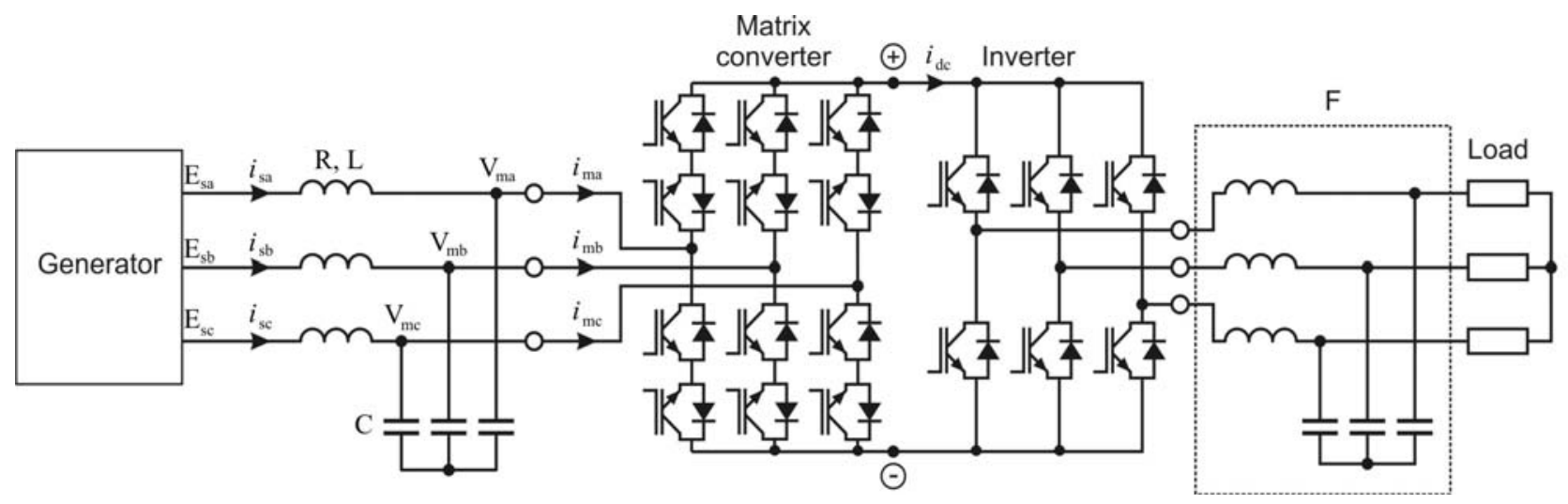

Fig. 14. Schematic diagram of the system with matrix converter.

\section{Conclusions}

The assumption for power-electronics converters was that the source of the electrical power in micropower station is high rotation speed generator with permanent magnets, described in chapter 2 . The generator support three-phase output voltage, which frequency and amplitude depend on rotation speed and construction. For the given generator construction the output power is a function of the rotation speed. It can be assumed that the generator output voltage frequency is not less than ten times higher than the power grid frequency $(50 \mathrm{~Hz}$ in Poland) and the voltage amplitude is in the range of low voltage $(<1000 \mathrm{~V})$. All the presented solutions are related to these assumptions.

In chapter 3 the three different solutions of the power-electronics converters have been presented. These solutions have different levels of complexity and different properties.

The first solution - converter with diode rectifier can be useful in micro-power plants of smallest scale, with power of $2 \div 3 \mathrm{~kW}$. This kind of converter introduce the number of harmonics into generator output currents. The effect of this is that useful output power is much less than reactive power of the generator. So the demand is that the generator for this kind of converter should have a considerable reserve of power in comparison to the required power plant output power. Further disadvantage is that the current distortions are transferred to the turbine blades, causing additional vibrations. Nonetheless it is the cheapest solution with the simplest control, so it should be considered in smallest appliances.

The converter with active filter presented in chapter 3.2 is considered as a solution for micro-power stations of power up to few hundreds of $\mathrm{kW}$. The use of active filter allows for significant reduction or elimination of the generator current harmonics, which increase the efficiency of power conversion and improves the working conditions of the turbine. But converter with active filter is more complex than the previous one and also needs a sophisticated control circuitry.

In chapters 3.3 and 3.4 the examples of control algorithms that can be applied for controlling the APF active filter have been presented. These algorithms give an overall view on the problems which should be solved for controller design of such structure. Because of the complexity of algorithms it is necessary in this case to use most powerful DSP processors. The rapid progress in control technique and development of the ultra-fast floating-point DSP processors made it possible to develop control systems for complex converter structures with input and/or output active filters. The main cost related to the control system is nowadays connected with developing the algorithm with adequate properties.

The matrix converter described in chapter 3.5 can be applied in biggest power stations with power in range 
500 and more $\mathrm{kW}$. This type of converter allows to achieve full control of the shape of the generator current waveforms and also control over system power factor in wide range of load values. These properties result in optimal use of the turbine and achieving high system efficiency. On the other hand it is the most expensive solution presented in the paper. Anyhow, because of its favorable properties and elimination of the electrolytic capacitors, this solution is worth considering as an alternative to converter with active filter.

The presented above power conversion circuits are not the only ones available. Other solutions like multilevel and resonant converters are also very promising and should be seriously considered. The research works on these kinds of converters are actually carried on in The Electrotechnical Institute. I.e. "backto-back" converter with multilevel inverters can prove to be the optimal solution for higher power plants. The useful mathematical models of multilevel inverters are described in [10].

The problems related to the protection and synchronization necessary in case of micro-power plant connection to the power grid have not been analyzed in the paper. The same limitation is also valid for connecting few micro-power plants to the common network. If the micro-power plant is designed only for generating the electrical power for supplying the separated home network than protection and synchronization appliances are not necessary. Also in this case it is possible to eliminate the output transformer.

Designing the circuitry for connecting the micropower plant into the power grid it is very important to make the economical analyze of the necessary powerelectronics devices and protection apparatus. In the literature is easy to find analyzes of different circuitry solutions also with economical analyzes. From these it can be derived that the economical factor cost $/ 1 \mathrm{~kW}$ is became more favorable with increasing the amount of produced energy. Especially for units with power higher than $250 \mathrm{kWh}$ the economical factor is much lower than for small units of power below $10 \mathrm{~kW}$. This relation is no surprise because the cost of power-electronic devices and protection apparatus is relatively lower and in the same time the system efficiency is relatively higher.

\section{Literature}

1. Mikielewicz D., Mikielewicz J.: "Micro-power plants: A novel application of cooling agents", Refrigeration \& Air-conditionig Engineering, 3/2008, pp. 98-104.

2. Murray W. Davis: "Mini Gas Turbines and High Speed Generators: a Preferred Choice for Serving Large Commercial Customers and Microgrids, Part I - Generating System", IEEE , pp. 669-676, 2002.

3. Murray W. Davis, Theodore J. Krupa and Arland Gifford: "Microturbines an Economic and Reliability Evaluation for Commercial, Residential, and Remote Load Applic.", IEEE Transactions on Power Systems, PE-480 PWR-0-10-1998. pp. 1-7.

4. Murray W. Davis: "Distributed Resource Power Systems Offer Significant Advantages Over Central Station Generation and T\&D Power Systems", IEEE T\&DConference and Exposition, Atlanta, Georgia, October 28 - November 1, 2001.

5. Fu-Sheng Pai, Shyh-Jier Huang: Design and Operation of Power Converter for Microturbine Powered Distributed Generator with Capacity Expansion Capability", IEEE Transactions on Energy Conversion, vol. 23, No.1, March 2008.

6. Haoran Bai, Fengxiang Wang, Tianyu Wang: "A SHEPWM Modulation Strategy of APF to Reduce Current Harmonics for High Speed PM Generator", Proceedings of International Conference on Electrical Machines and Systems 2007, October 811, Seoul, Korea.

7. Hamouda M., AL-Haddad K., Fnaiech F., Blanchette H.: "Input-State Feedback Linearization Control of Two-Stage Matrix Converters Interfaced with High-Speed Microturbine Generators”, 2007 IEEE Canada Electrical Power Conference.

8. Iwaszkiewicz J.: "A Predictive Algorithm for Current Controlled Voltage Source Inverter", IEEE International Symposium on Industrial Electronics, ISIE 93, Budapest, 1993.

9. Perz J.: „An active filter control for improvement of the voltage inverter output current waveforms", $\mathrm{PhD}$ thesis, The Electrotechnical Institute, 2000.

10. Iwaszkiewicz J.: „Mathematical models of powerelectronics multilevel converters - analysis and applications", Proceedings of Electrotechnical Institute, no. 227, monograph, pp.142, 2006. 
